\title{
ON THE WESS-ZUMINO-WITTEN ANOMALOUS FUNCTIONAL AT FINITE TEMPERATURE
}

\author{
R.F.Alvarez-Estrada, A. Dobado and A.Gómez Nicola \\ Departamento de Física Teórica, Universidad Complutense \\ 28040, Madrid, Spain
}

July 12, 2011

\begin{abstract}
We discuss the finite temperature extension of the anomalous Wess-ZuminoWitten lagrangian. The finite temperature $S^{1} \times S^{3}$ compactification makes a structure in disconnected sectors, corresponding to different baryon numbers appear naturally. The consistency of the anomalous functional is proved for arbitrary baryon number configurations. The anomalous behavior of the functional is shown to be consistent with the absence of finite temperature corrections to chiral anomalies in QCD, for each baryon number sector.

$\mathrm{FT} / \mathrm{UCM} / 9 / 93$
\end{abstract}


The low-energy properties of QCD have been successfully studied in the framework of the effective lagrangian method [1] [2], also called Chiral Perturbation Theory $(\chi \mathrm{PT})$. In this formalism, the anomalous sector of the theory at zero temperature (ZT) is described through the inclusion of the Wess-Zumino-Witten (WZW) lagrangian [3] 园] (see also [5] for a complete and detailed exposition), which reproduces the correct QCD anomalies and, consequently, the low-energy limit of anomalous processes involving hadrons and photons, such as $K^{+} K^{-} \rightarrow \pi^{+} \pi^{-} \pi^{0}$ or $\pi^{0} \rightarrow \gamma \gamma$. The ZT WZW action cannot be expressed as a local four-dimensional integral, but it has to be performed on a fivedimensional space whose boundary is the standard four-dimensional space-time. $\chi \mathrm{PT}$ can also be studied in the finite temperature $(T \neq 0)$ case, corresponding to a hadronic medium in thermal equilibrium at temperature $T$. Thermodynamical quantities, like the pressure and the behavior of the quark condensate, have been analyzed in great detail in [6] for low temperatures (yet excluding anomalous WZW effects). Analogous studies with effective models have been carried out in [7]. In addition to that, one may well want to study the anomalous processes listed above, in the presence of the medium. Then, a suitable extension of the WZW anomalous lagrangian to the $T \neq 0$ case is needed. In the $T=0$ case, one can compactify the euclidean space-time to the four dimensional sphere $S^{4}$. Then, the Goldstone fields for the $N_{f}=3$ case ( $N_{f}$ being the number of flavours) are mappings $U(x): S^{4} \rightarrow S U(3)$ ( $x$ being in $S^{4}$ ). The lagrangian to the lowest order in the number of derivatives, and without any gauge coupling, is then given by the non-linear 
sigma-model one plus the WZW term [4] [5]:

$$
\Gamma[U]=\frac{1}{4} F^{2} \int_{S^{4}} d x \operatorname{Tr}\left\{\partial_{\mu} U^{-1} \partial^{\mu} U-M^{2}\left(U+U^{-1}\right)\right\}+\frac{N_{c}}{240 \pi^{2}} \int_{I \times S^{4}} \operatorname{Tr}\left(U_{t}^{-1}\left(d+d_{t}\right) U_{t}\right)^{5}
$$

Here, $F \simeq 93 \mathrm{MeV}$ is the pion decay constant, $M$ is the mass matrix, $N_{c}$ is the number of colours, $I$ is the interval $[0,1]$ in which $t$ varies , $d \equiv \partial_{\mu} d x^{\mu}, d_{t} \equiv \partial_{t} d t$. The last term is antisymmetrized in all the indices, according to the notations in [5], which we shall use in what follows almost everywhere. $U_{t}$ is a continuous mapping $U_{t}(x): I \times S^{4} \rightarrow S U(3)$ such that $U_{0}(x)=1, U_{1}(x)=U(x)$ i.e. it is a homotopy. Since the fourth group of homotopy $\pi_{4}(S U(3))=0$, all the mappings $U(x)$ are deformable one into another, and such an homotopy $U_{t}(x)$ always exists for any $U(x)$. The WZW term appearing in eq.(1) is independent of the homotopy $U_{t}(x)$ and, so, it is in fact a function of the chiral field $U(x)$ only.

In the $T \neq 0$ case, the situation is quite different : in the imaginary-time formalism for the partition function [8], the euclidean time coordinate $\tau$ runs from 0 to $\beta=1 / T$ and the fields are periodic in $\tau(U(\vec{x}, \tau+\beta)=U(\vec{x}, \tau))$. Then, if we compactify the euclidean three-space to $S^{3}$ by imposing boundary conditions at infinity, we deal with applications $U(\vec{x}, \tau): S^{1} \times S^{3} \rightarrow S U(3) . \vec{x}$ will always denote a vector in $S^{3}$. These applications are not all homotopically equivalent, i.e. they are not deformable into one another as in the previous case of the $S^{4}$ compactification at $T=0$. In fact, there are as many homotopy classes of those mappings as integer numbers [9]. The integer label that classifies a given 
map can be written as:

$$
N_{B}=\frac{1}{24 \pi^{2}} \int_{S^{3}}\left(U^{-1}(\vec{x}, \tau) \overrightarrow{d U}(\vec{x}, \tau)\right)^{3}
$$

where $\vec{d} \equiv \vec{\partial} d \vec{x}$ in three dimensions and (目) is antisymmetric in all the spatial indices. Notice that $N_{B}$ in (2) for a given $\tau$ is the winding number in $\pi_{3}(S U(3))$. As $\pi_{3}(S U(3))=\mathcal{Z}$ (the set of all integers) also, $N_{B}$ cannot depend on $\tau$, and it in fact characterizes topologically different maps in $S^{1} \times S^{3}$. This integer $N_{B}$ has a natural physical interpretation as the baryon number of the configuration.

In this work, we shall consider the case in which weak gauge couplings are neglected. Then, baryon number is strictly conserved. Under these conditions, there exist different disconnected sectors corresponding to $N_{B}=0, \pm 1, \pm 2, \ldots$. Transitions between them are forbidden, and we must define our WZW lagrangian in each sector separately, because there is not any homotopy transformation connecting every $U(\vec{x}, \tau)$ field with the identity. If gauge couplings with $S U(2)_{L}$ gauge fields were considered, the baryon number current would become anomalous [10] and transitions from one sector to another would in principle be allowed.

At this point, we would like to stress that the $S^{1} \times S^{3}$ compactification scheme is useful not only to study finite temperature effects, but also at ZT in cases where it is important to have a clear realization of the baryon number 四.

Let us consider now the following $S^{1} \times S^{3}$ WZW functional at finite temperature for 
the $j$-th $\left(\right.$ or $\left.N_{B}=j\right)$ sector:

$$
\Gamma_{j}\left[U(\vec{x}, \tau) ; \bar{U}_{j}(\vec{x}, \tau)\right]=N \int_{I \times S^{1} \times S^{3}} \operatorname{Tr}\left(U_{t}^{-1}(\vec{x}, \tau)\left(d+d_{t}\right) U_{t}(\vec{x}, \tau)\right)^{5}
$$

$U(\vec{x}, \tau)$ being in the $j$-th sector. $\bar{U}_{j}(\vec{x}, \tau)$ is a given field also belonging to the $j$-th sector and hence, in the general case, it is different from the identity, except for $j=0$ where it can be taken to be unity $\left(\bar{U}_{0}(\vec{x}, \tau)=1\right)$. $U_{t}(t \in I)$ is now a continuous application $U_{t}(\vec{x}, \tau): I \times S^{1} \times S^{3} \rightarrow S U(3)$ connecting the chiral field $U(\vec{x}, \tau)=U_{1}(\vec{x}, \tau)$ and the homotopy class representative, $\bar{U}_{j}(\vec{x}, \tau)=U_{0}(\vec{x}, \tau) . \quad N$ is a constant to be determined. As in the $T=0$ case, in order to show that $\Gamma_{j}$ is the correct anomalous functional, it is necessary to demonstrate that it is independent of the choice of the homotopy operator $U_{t}(\vec{x}, \tau)$, i.e., it depends only of the values at the boundary, and that it reproduces the QCD anomalies (for instance, the $U_{A}(1)$ anomaly that gives rise to the $\pi^{0} \rightarrow \gamma \gamma$ reaction).

Furthermore, in this case, it is necessary to prove also the independence of the results on the choice of the class representative $\bar{U}_{j}(\vec{x}, \tau)$.

In order to show the independence of the functional in eq.(3) on the homotopy connecting the chiral field with the homotopy class representative, we consider the difference $\Gamma_{j}\left[U_{t}^{\prime}\right]-\Gamma_{j}\left[U_{t}\right] . \quad U_{t}^{\prime}$ and $U_{t}$ are two different mappings satisfying the required boundary conditions, say, at $t=0,1$ they both are equal to $\bar{U}_{j}(\vec{x}, \tau), U(\vec{x}, \tau)$ respectively. $\Gamma_{j}\left[U_{t}\right]$ is just the right-hand-side (r.h.s.) of eq.(3) and so on for $\Gamma_{j}\left[U_{t}^{\prime}\right]$. By using the transformation formula [5]:

$$
\operatorname{Tr}\left\{(g h)^{-1}\left(d+d_{t}\right)(g h)\right\}^{5}=\operatorname{Tr}\left(g^{-1}\left(d+d_{t}\right) g\right)^{5}+\operatorname{Tr}\left(h^{-1}\left(d+d_{t}\right) h\right)^{5}+\left(d+d_{t}\right) \alpha(g, h)
$$


where $g$ and $h$ are applications from a five-dimensional manifold into the $S U(3)$ group and $\alpha(g, h)$ is a 4 -form whose detailed form can also be found in [5], it is possible to write:

$$
\Gamma_{j}\left[U_{t}^{\prime}\right]-\Gamma_{j}\left[U_{t}\right]=N \int_{S^{1} \times S^{1} \times S^{3}} \operatorname{Tr}\left(h_{t}^{-1}(\vec{x}, \tau)\left(d+d_{t}\right) h_{t}(\vec{x}, \tau)\right)^{5}
$$

with $h_{t}(\vec{x}, \tau)=U_{t}^{\prime}(\vec{x}, \tau) U_{t}^{-1}(\vec{x}, \tau)$. The $\alpha$ term vanishes due to the property $\alpha(g, 1)=$ $\alpha(1, g)=0$ for any $g$. $h_{t}$ is periodic in $t$, and so the above difference is equivalent to compactify the $I$ interval into $S^{1}$ (by identifying the extreme points).

If we want our quantum partition function to be insensitive to the above variation, the difference in (5) must be $2 \pi i n, n$ being an integer. This is almost trivial in the $T=0$ case, because the integral in (5) is over $S^{1} \times S^{4}$ and mappings of this space into $S U(3)$ are classified by the homotopy group $\pi_{5}(S U(3))=\mathcal{Z}$ [9]. But (5), when integrated over $S^{1} \times S^{4}$, is just the expression for the winding number in $\pi_{5}(S U(3))$ and so, it is equal to $2 \pi$ in if the proper choice of the constant $N$ is made. However, in our present case things are different. The mappings from $S^{1} \times S^{1} \times S^{3}$ into $S U(3)$ are not classified by $\pi_{5}(S U(3))$ but there are $\mathcal{Z} \times \mathcal{Z}$ classes [9]. Hence, in principle, it is not clear whether (5) should be $2 \pi n i$.

To prove that this is really the case, we make use of the Atiyah-Singer index theorem [11] in six dimensions, which reads [5] :

$$
\text { ind } i \not D[A]=\frac{-i}{48 \pi^{3}} \int_{M_{6}} \operatorname{Tr} F^{3}
$$

Here, $i \not D$ is the usual Dirac operator for fermions coupled to a $S U(3)$ gauge field $A$ with strength field $F=d A+A^{2}$, and $M_{6}$ is any six-dimensional Euclidean and compact 
manifold. ind $i \not D$ is the index of the Dirac operator and it is, of course, an integer. Let us consider the space $M_{6}=S^{1} \times S^{1} \times S^{4}$, which can be decomposed as the union of eight patches isomorphic to $\mathcal{R}^{6}$, namely $I^{ \pm} \times I^{ \pm} \times D_{4}^{ \pm}$, where $D_{4}$ is a four-dimensional disk and $I$ is the unit interval. A given gauge field is only well defined over each patch (for example $\left.I^{+} \times I^{+} \times D_{4}^{-}\right)$. Two gauge fields $A_{1}, A_{2}$, corresponding to two different patches, must differ by a gauge transformation defined on the intersection of the patches i.e. $A_{2}=g^{-1}\left(A_{1}+d\right) g$, $g$ being an application from the intersection of the corresponding patches into $S U(3)$. Let us consider the following gauge field configuration : $A^{ \pm \pm+}=g^{-1} d g ; A^{ \pm \pm-}=0$ where $g$ is an arbitrary map $g(x): S^{1} \times S^{1} \times S^{3} \rightarrow S U(3)$. The superscripts in the $A^{\prime}$ 's fields denote the patches in which those fields are defined. It can be checked that this is a well defined gauge field, since the appropiate consistency relations are fulfilled by the transition functions on all intersections of three or more patches. By definition of the Chern-Simons form $Q_{5}[A(x), F(x)]$ [5], $x$ being now in $M_{6}$, we have over any single patch $\operatorname{Tr} F^{3}=d Q_{5}[A, F]$ (note that this is not true over the whole manifold). Furthermore, $Q_{5}[0,0]=0$ and $Q_{5}\left[g^{-1} d g, 0\right]=\frac{1}{10} \int_{S^{1} \times S^{1} \times S^{3}} \operatorname{Tr}\left(g^{-1} d g\right)^{5}$. Then, by writing the r.h.s. of (6) for the above gauge field configuration as a sum over the different patches and, then, applying the Stokes theorem in each patch, we arrive to the desired result, namely:

$$
\frac{\Gamma_{j}\left[U_{t}^{\prime}\right]-\Gamma_{j}\left[U_{t}\right]}{2 \pi i} \in \mathcal{Z} \quad \text { if } \quad N=\frac{m}{240 \pi^{2}}
$$

$m$ being an integer. Another way to prove this is to take $M_{6}=S^{2} \times S^{4}=\sum D_{2}^{ \pm} \times D_{4}^{ \pm}$ and the gauge field configuration $A^{ \pm+}=g^{-1} d g ; A^{ \pm-}=0$ with $g(x): S^{2} \times S^{3} \rightarrow S U(3)$, with the restriction that $g$ takes the same value in the two poles of $S^{2}$. Then, it is 
equivalent to an arbitrary map of $S^{1} \times S^{1} \times S^{3}$ into $S U(3)$. Notice that the result (17) is independent of the choice of class representative $\bar{U}_{j}(\vec{x}, \tau)$.

As discussed above, the next thing we have to study is the transformation properties of the functional in eq.(3). In order to do that, we consider two arbitrary representatives of the $j$-th class $\bar{U}_{j}^{A}(\vec{x}, \tau)$ and $\bar{U}_{j}^{B}(\vec{x}, \tau)$. For any chiral field $U(\vec{x}, \tau)$ belonging to the $j$-th class there exist homotopies $U_{t}^{A}(\vec{x}, \tau)$ and $U_{t}^{B}(\vec{x}, \tau)$ interpolating between the chiral field and $\bar{U}_{j}^{A}(\vec{x}, \tau)$ and $\bar{U}_{j}^{B}(\vec{x}, \tau)$ respectively, so that $U_{0}^{A}=\bar{U}_{j}^{A}, U_{1}^{A}=U, U_{0}^{B}=\bar{U}_{j}^{B}$ and $U_{1}^{B}=U$. It is also clear that $\bar{U}_{j}^{A-1} \bar{U}_{j}^{B}$ belongs to the trivial class or, in other words, there is one homotopy $\omega_{t}$ connecting $\bar{U}_{j}^{A-1} \bar{U}_{j}^{B}$ with the identity and, then, we have $\omega_{0}=\bar{U}_{j}^{A-1} \bar{U}_{j}^{B}$ and $\omega_{1}=1$

Now we consider the expression (丑) for the change of the Chern-Simons form for a pure gauge field under a gauge transformation, and we apply this formula to the particular case where $g=U_{t}^{A}$ and $h=\omega_{t}$, together with the definition of our functional in eq.(3) $\Gamma_{j}\left[U_{t}\right]=\Gamma_{j}\left[U ; \bar{U}_{j}\right]$. We find:

$$
\Gamma_{j}\left[U_{t}^{A} \omega_{t}\right]=\Gamma_{j}\left[U_{t}^{A}\right]+\Gamma_{j}\left[\omega_{t}\right]+\int_{I \times S^{1} \times S^{3}}\left(d+d_{t}\right) \alpha\left(U_{t}^{A}, \omega_{t}\right)
$$

As it was discussed above, the functional $\Gamma_{j}\left[U_{t}\right]$ depends only on the values that $U_{t}$ takes at $t=0$ and $t=1$ and, in addition, $U_{t}^{A} \omega_{t}$ is equal to $U_{t}^{B}$ at $t=0$ and $t=1$. Then, we can write:

$$
\Gamma_{j}\left[U_{t}^{A} \omega_{t}\right]=\Gamma_{j}\left[U_{t}^{B}\right]=\Gamma_{j}\left[U ; \bar{U}_{j}^{B}\right]
$$

We also have:

$$
\Gamma_{j}\left[U_{t}^{A}\right]=\Gamma_{j}\left[U ; \bar{U}_{j}^{A}\right] ; \quad \Gamma_{j}\left[\omega_{t}\right]=-\Gamma_{j}\left[\bar{U}_{j}^{A-1} \bar{U}_{j}^{B} ; 1\right]
$$


So, we can write:

$$
\Gamma_{j}\left[U ; \bar{U}_{j}^{B}\right]=\Gamma_{j}\left[U ; \bar{U}_{j}^{A}\right]-\Gamma_{j}\left[\bar{U}_{j}^{A-1} \bar{U}_{j}^{B} ; 1\right]-\alpha\left(\bar{U}_{j}^{A}, \bar{U}_{j}^{A-1} d \bar{U}_{j}^{B}\right)
$$

where $\alpha(g, 1)=0$ has again been used. Therefore, we arrive to the conclusion that $\Gamma_{j}\left[U ; \bar{U}_{j}^{B}\right]-\Gamma_{j}\left[U, \bar{U}_{j}^{A}\right]$ does not depend on $U$. Thus, if we introduce another chiral field $U^{\prime}$ belonging also to the $j$-th class, we get:

$$
\Gamma_{j}\left[U^{\prime} ; \bar{U}_{j}^{B}\right]-\Gamma_{j}\left[U ; \bar{U}_{j}^{B}\right]=\Gamma_{j}\left[U^{\prime} ; \bar{U}_{j}^{A}\right]-\Gamma_{j}\left[U ; \bar{U}_{j}^{A}\right]
$$

This is a very interesting result, since it shows that the variations of the functional in eq.(3) corresponding to small variations (in the topological sense) of the chiral field $U$ do not depend at all on the class representative $\bar{U}_{j}$. This applies of course also to the anomalous variations or the Green functions obtained from this functional. The independence of Green functions on $\bar{U}_{j}$ can be seen, for instance, by expanding $\Gamma_{j}\left[U ; \bar{U}_{j}\right]$ near a classical field configuration $U=U_{c}$ in order to get the effective action including loops, and by taking into account eq.(12). As a particular case, we can consider the trivial sector $j=0$ and choose the identity as the class representative. Now, we can write the chiral field $U$ in terms of the Goldstone boson fields $\pi_{a}$ in the standard way as $U=\exp \left(i \sum_{a} \lambda_{a} \pi_{a} / F\right)$. Then it is possible to make a derivative expansion in eq.(3) retaining only the lowest (four) derivatives. Finally, by using the Stokes theorem, we get an action which is the integral on $S^{1} \times S^{3}$ of a lagrangian which properly reproduces processes like $K^{+} K^{-} \rightarrow \pi^{+} \pi^{-} \pi^{0}$.

Now we want to see that the functional in eq.(3) reproduces the QCD anomalies and, in particular, the $U_{A}(1)$ anomaly responsible for the $\pi^{0} \rightarrow \gamma \gamma$ decay. In order to show 
that, let us gauge $\Gamma_{j}\left[U ; \bar{U}_{j}\right]$ with the electromagnetic field $A_{\mu}$, to make it invariant under infinitesimal local charge transformations $U(\vec{x}, \tau) \rightarrow U(\vec{x}, \tau)+i \varepsilon(\vec{x}, \tau)[Q, U(\vec{x}, \tau)] \equiv U^{\prime}$ with $Q$ the quark charge matrix. We start from $\Gamma_{j}\left[U^{\prime} ; \bar{U}_{j}\right]$ written in (3) in terms of $U_{t}^{\prime}$ such that $U_{0}^{\prime}=\bar{U}_{j}(\vec{x}, \tau)$ and $U_{1}^{\prime}=U^{\prime}(\vec{x}, \tau)$. But $\Gamma_{j}\left[U_{t}^{\prime}\right]=\Gamma_{j}\left[U_{t} \omega_{t}\right]$ with $U_{0}=\bar{U}_{j}, U_{1}=U$, $\omega_{0}=1, \omega_{1}=1+v$ and $v=i \varepsilon\left(U^{-1} Q U-Q\right)$ and so, by applying again (4) we obtain :

$$
\Gamma_{j}\left[U^{\prime} ; \bar{U}_{j}\right]-\Gamma_{j}\left[U ; \bar{U}_{j}\right]=\Gamma_{j}[1+v ; 1]+\alpha(U, 1+v)
$$

where the $\alpha$ term is four-dimensional and it is the $t=1$ contribution.

Again the $t=0$ one vanishes $(\alpha(g, 1)=0)$. As we have seen in the general case in eq.(12), the variation of $\Gamma_{j}$ has always the same form for each sector, independently of $\bar{U}_{j}$. On the other hand, the r.h.s of (13) when expanded in $\varepsilon(x)$ is the four-dimensional $T=0$ part [4] with the replacement $S^{4} \rightarrow S^{1} \times S^{3}$. Then, the gauged WZW functional has the same dependence on $A_{\mu}$ for every sector. The same argument applies if we introduce an external $U_{A}(1)$ field $a_{\mu}$ to derive the axial current in the usual way as $J_{\mu}^{A}=\frac{\delta \Gamma_{j}\left[U, A_{\mu}, a_{\mu} ; \bar{U}_{j}\right]}{\delta a_{\mu}}$. As the dependence of the gauged functional $\Gamma_{j}\left[U, A_{\mu}, a_{\mu} ; \bar{U}_{j}\right]$ on $a_{\mu}$ is the same for each sector, $J_{\mu}^{A}$ has the same form in terms of each $U$ field, which turns out to be also the $T=0$ form with the replacement commented above. Then, the anomaly for $\partial^{\mu} J_{\mu}^{A}$ when $T \neq 0$ remains the same as in QCD for $T=0$ in terms of the $A_{\mu}$ field by choosing $m=N_{c}$, with the above replacement. Therefore, it has been shown that the WZW term in eq.(3) is consistent with other previous results on QCD anomalies at finite temperature 12.

As a consequence of the above discussion, we arrive at the conclusion that the functional, in eq.(3) is the proper generalization of the WZW functional when one uses the 
compactification $S^{1} \times S^{3}$ instead of the original $S^{4}$. The $S^{1} \times S^{3}$ compactification seems to be more appropriate to study either finite temperature effects, or configurations with baryon number different from zero or both simultaneously. However, one could still ask about the role that the class representative $\bar{U}_{j}$ plays in the definition of the functional in eq.(33). This functional effectively depends on $\bar{U}_{j}$ but, as we have shown in this work, the variation $\delta \Gamma_{j}\left[U ; \bar{U}_{j}\right]$ produced by a variation in the chiral field $\delta U$ is independent on the class representative $\bar{U}_{j}$. Then, all contributions to the Goldstone boson Green functions coming from the functional in eq.(3) do not depend at all on $\bar{U}_{j}$. Thus the particular choice of the class representative has no effect on observables such as widths or crosssections. In some sense. the class representative is as irrelevant as the value of a constant added to the interaction lagrangian of a $\lambda \Phi^{4}$ theory.

Following this analogy, and noting that $\Gamma_{j}[U ; U]=0$, one could think that a natural choice for the class representative $\bar{U}_{j}$ could be some static field configuration minimizing the energy, i.e., representing the vacuum of this sector or, in other words, representing the state of the theory with minimal energy for fixed baryon number $N_{B}=j$. For example, for $j=0$ this state would be the QCD vacuum which corresponds to $\bar{U}_{0}=1$, thus recovering the standard WZW functional (but with different boundary conditions for the chiral field and with the $T$-dependence in the $T \neq 0$ case). Let us consider now the case of the non-trivial sectors. For instance, we can consider the $j=1$ class. Obviously, the state of minimal energy with baryon number equal to one should describe the nucleon. However, a nucleon can be located at any place of space and, therefore, we do not have a 
single state but many of them, degenerated and connected by simple spatial translations. The description of these states in terms of chiral fields is, clearly, some kind of skyrmion configuration located in some given point of the space. However, to obtain the concrete form of this configuration one needs to compute the effect of the higher derivative terms appearing in the $\chi P T$ lagrangian. It is clear that, in practice, this can only be achieved in an approximate way, for instance, by retaining only a few of the lowest derivative terms. Once an appropriate description of the chiral field describing the nucleon be available, the functional in eq.(3) could be used to compute anomalous reactions in the $j=1$ sector, such as the decay of pseudoscalar excitations of the nucleon into two photons and one non-excited proton. Of course, this discussion can be extended to the low energy states corresponding to higher baryon numbers, which could in principle provide a description of other nuclei.

One may also calculate the effects of loops in $\chi \mathrm{PT}$ in the trivial sector, by taking into account the functional of eq.(3). The compactification $S^{1} \times S^{3}$ allows us to carry out these calculations at finite temperature and/or finite volume (the total action for computing loops is now the obvious finite temperature extension of the first integral in the r.h.s. of (1i) plus (3)). For instance, it is possible to obtain non trivial finite- $T$ corrections to the decay of the $\pi^{0}$ into two photons [13]. This is important in a theoretical sense, because one must check that there are no infinite contributions that would change the constant $N$ in (7) due to renormalization effects. Also, from a phenomenological point of view, the $\pi^{0}$ could be living in a hadronic medium and temperature effects affecting its decay can 
be analyzed. It is important to recall, that in the finite volume case, one must take into account the fact that the chiral limit $M \rightarrow 0$ and the infinite volume one $V \rightarrow \infty, V$ being the volume, do not conmute (see [14]). At finite $V$ there is no spontaneous symmetry breaking, and the limit $M \rightarrow 0$ is inconsistent. The symmetry must be explicitely broken with the mass term and then the infinite volume limit can be taken. This is a crucial point here, because the $R \rightarrow \infty$ limit, $R$ being the radius of $S^{3}$, must be taken at some stage. This means, of course, that our actual extension of the WZW functional allows to study in general the $T \neq 0$ case with infinite volume.

To conclude, we would like to summarize the main results of this work: The following functional :

$$
\begin{array}{r}
\Gamma_{j}\left[U, \bar{U}_{j}\right]=\frac{N_{c}}{240 \pi^{2}} \int_{0}^{1} d t \int_{S^{1} \times S^{3}} \epsilon^{i j k l m} U_{t}^{-1} \partial_{i} U_{t} U_{t}^{-1} \partial_{j} U_{t} U_{t}^{-1} \partial_{k} U_{t} U_{t}^{-1} \partial_{l} U_{t} U_{t}^{-1} \partial_{m} U_{t} \\
U_{0}=\bar{U}_{j}(\vec{x}, \tau) \quad U_{1}=U(\vec{x}, \tau) \quad i \ldots m=1, \ldots, 5
\end{array}
$$

which is a more explicit form of (3), provides the proper version of the WZW functional for $S^{1} \times S^{3}$ compactification of the space time reproducing correctly the QCD anomalies. This compactification is appropriate for finite temperature or/and finite volume computations, so that we can reproduce previous results in QCD anomalies and study processes like $\pi^{0} \rightarrow \gamma \gamma$, both at finite $T$ and/or finite $V$. It also provides a clear description of the baryon number in $\chi P T$. In particular, the functional in eq.(14) (or its gauged version with the electromagnetic field) could in principle be used to compute anomalous processes involving states with non zero baryon number. One could also envisage the possibility of 
gauging the $S U(2)_{L}$ group. In this case, the baryon number becomes anomalous and it is not conserved any more, specially at very high temperatures. However, the discussion in this case is more involved and it will be presented elsewhere.

We are grateful to L.A.Ibort for some discussions and help. The financial support of C.I.C.Y.T (Projects AEN90-0034 and AEN93-0776), Spain, is acknowledged. 


\section{References}

[1] S.Weinberg, Phys.Rev.166 (1968) 1568; Physica A96 (1979) 327.

[2] J.Gasser and H.Leutwyler, Ann.Phys. 158 (1984) 42 ; Nucl.Phys.B307 (1988) 763.

[3] J.Wess and B.Zumino, Phys.Lett 37B (1971) 95.

[4] E.Witten, Nucl.Phys.B223 (1983) 422; B223 (1983) 433.

[5] L.Alvarez-Gaumé and P.Ginsparg, Ann.Phys. 161 (1985) 423.

[6] P.Gerber and H.Leutwyler, Nucl.Phys B321 (1989) 387.

[7] A.Barducci, R.Casalbuoni, S.de Curtis, R.Gatto and G.Pettini, Phys.Rev D46 (1992) 2203.

[8] N.P.Landsman and Ch.G.van Weert, Phys.Rep.145 (1987) 141.

[9] G.W.Whitehead : "Elements of Homotopy Theory" (Springer-Verlag, 1978).

[10] G’t Hooft Phys.Rev.Lett.37 (1976) 8;Phys.Rev D14 (1976) 3432.

[11] M.F.Atiyah and I.M.Singer, Ann.of Math 87 (1968) 485,546; 93 (1971) 119,139.

[12] R.F.Alvarez Estrada in "Thermal Field Theories", eds. H.Ezawa and T.Arimitsu (North Holland, Amsterdam, 1991) p.423; A.V.Smilga, Phys.Rev.D45 (1992) 1378;C.Contreras and M.Loewe, Zeit.Phys.C.Part.Fields. 40 (1988) 253; Y.L.Niu and G.J.Ni. Phys.Rev.D38 (1988) 3840; L.Dolan and R.Jackiw, Phys.Rev. D9 (1974) 
3320; A.Das and A.Karev, Phys.Rev.D36 (1987) 623; R.Baier and E.Pilon, Z.Phys.C. 52 (1991) 339; H.Itoyama and A.H.Mueller, Nucl.Phys.B218 (1983) 349.

[13] A.Dobado, R.F.Alvarez-Estrada and A.Gomez Nicola, work in preparation.

[14] J.Gasser and H.Leutwyler, Phys.Lett.B188 (1987) 477; H.Leutwyler, Phys.Lett.B189 (1987) 197. 